\title{
A prohibition of equilibrium spin currents in multi-terminal ballistic devices
}

\author{
A. A. Kiselev and K. W. Kim \\ Department of Electrical and Computer Engineering, North Carolina State University, Raleigh, NC 27695-7911
}

\begin{abstract}
We show that in the multi-terminal ballistic devices with intrinsic spin-orbit interaction connected to normal metal contacts there are no equilibrium spin currents present at any given electron energy. Obviously, this statement holds also after the integration over all occupied states. Based on the proof of this fact, a number of scenarios involving nonequilibrium spin currents is identified and further analyzed. In particular, it is shown that an arbitrary two-terminal device cannot polarize transient current. The same is true for the output terminal of an $N$-terminal device when all $N-1$ inputs are connected in parallel.
\end{abstract}

PACS numbers: 73.63.-b; 72.25.-b

The results of Murakami et al. ${ }^{1}$ and Sinova et al. ${ }^{2}$ excited spintronic community by suggesting presence of the dissipationless spin currents in electron/hole systems with intrinsic spin-orbit (SO) interaction. A quick and massive response by quite a few of the research teams approached varied levels of generality and with mixed conclusions. $^{3}$

The matter was further highlighted by Rashba ${ }^{4}$ who distilled the problem of equilibrium dissipationless spin currents in a two-dimensional (2D) electron gas into an unobscured puristic form of a paradox.

A similar phenomenology could potentially exist in 1D systems, especially in relation to the complex multiterminal ballistic devices. Rather obvious arguments (see, e.g., Ref. 5), although, put a ban on the existence of equilibrium spin currents in an ideal 1D wire with parabolic dispersion and linear-in- $k$ SO coupling. Nevertheless, for the 1D structures with static scatters, the opposite conclusions were recently announced ${ }^{6}$ (but, again, their validity is now also questioned ${ }^{7}$ ).

In this Report we provide an unambiguous proof of the fact that in multi-terminal ballistic devices equilibrium spin currents do not exist. This conclusion is universal and does not depend on the particular choice of the electron Hamiltonian or the particular type of the SO interaction.

We consider a ballistic device consisting of a structure of complex shape (for a simple example, some area, "stamped out" from the two-dimensional electron gas) that is connected to the exterior by a number of quasi1D wires (arms) - see Fig. 1 (a) for a visual guide.

To analyze the static linear-response conductances of the multi-terminal ballistic device we apply the Landauer-Büttiker formalism, ${ }^{8}$ that we have extended to incorporate also spin-related phenomena. ${ }^{9,10}$ Following closely these earlier publications, we now briefly introduce relevant basic concepts.

A finite-size scattering matrix $\mathbf{S},{ }^{8}$

$$
\mathbf{S}=\left(\begin{array}{cccc}
r_{11} & t_{12} & \cdots & t_{1 N} \\
t_{21} & r_{22} & \cdots & t_{2 N} \\
\vdots & \vdots & \ddots & \vdots \\
t_{N 1} & t_{N 2} & \cdots & r_{N N}
\end{array}\right)
$$

describes in a condensed form transmission and reflection properties of an arbitrarily complex linear (ballistic) system connected to the exterior via $1 \mathrm{D}$ wires. It consists of the (diagonal) reflection coefficients $r_{i i}$ (in the $i$-th channel) and (off-diagonal) transmission coefficients $t_{i j}$ (describing propagation of the particle from the $j$-th into the $i$-th channel, $i \neq j$ ). For every electron energy we choose to enumerate as distinguishable channels (and terminals) all energetically allowed electron fluxes through different 1D subbands (transverse modes), even in the same wire. Thus, the number of the channels can differ substantially from the number of the attached contacts. Because of this fact, biases applied to different terminals not always can be modulated independently. Since an elementary electron flux $F$ carried by a particular ideal 1D channel is defined by product of square of the electron plane wave amplitude $\psi$ and the state group velocity $v=\partial H / \partial k$, it is a usual practice to define the scattering matrix in terms of the current amplitudes $u=\sqrt{v} \psi$. Thus, $F \propto u^{+} u$.

For a spinless particle, the coefficients $r_{i i}, t_{i j}$ are scalars. By taking electron spin into consideration, the number of transmission channels effectively doubles. For this discussion, we will assume that in the vicinity of the normal metal contacts the channel asymmetry is either not present or effectively screened by the metal. Thus, the spin-dependent interactions are spatially limited to the interior of the system, and the transport in the leads through two degenerate spin subchannels is coherent and requires some cautious treatment, especially when evaluating total channel fluxes and their polarization.

To incorporate into our formalism this spin-related channel-doubling, we convert transmission and reflection coefficients of the $\mathbf{S}$ into $2 \times 2$ submatrices. Each $2 \times 2$ submatrix $\hat{x}\left(\hat{x}=\hat{r}_{i i}\right.$ or $\left.\hat{t}_{i j}\right)$ in this case can be equivalently expanded as

$$
\hat{x}=\left(\begin{array}{cc}
x_{\uparrow \uparrow} & x_{\uparrow \downarrow} \\
x_{\downarrow \uparrow} & x_{\downarrow \downarrow}
\end{array}\right)=\hat{1} x_{1}+i \sum_{\alpha} \hat{\sigma}_{\alpha} x_{\alpha}
$$

with a small additional benefit of conventional algebraic manipulations with matrix objects. Here $\hat{1}$ is a unit $2 \times$ 2 matrix, $\hat{\sigma}_{\alpha}(\alpha=x, y, z)$ are the usual Pauli matrices 
( $\uparrow$ and $\downarrow$ utilized here are eigenstates of the operator $\hat{\sigma}_{z}$, with axis $z$ actually arbitrarily chosen).

In case of degenerate spin subchannels, an elementary channel flux $F \propto \hat{u}^{+} \hat{u}$ (input or output) is conveniently given by the spinor column $\hat{u}=\left(u_{\uparrow}, u_{\downarrow}\right)$.

The relative magnitude and phase between $u_{\uparrow}$ and $u_{\downarrow}$ characterize orientation of the spin; vector $\boldsymbol{P}$, defined by three components $P_{\alpha}$ in the $x y z$ coordinate system, is called the polarization vector, $P_{\alpha} F \propto \hat{u}^{+} \hat{\sigma}_{\alpha} \hat{u}$. For the arbitrary spinor $\hat{u}$ the absolute value $|\boldsymbol{P}|=1$. When the incident flux $\hat{u}$ is pumped into the channel $j$, the $\hat{t}_{i j} \hat{u}$ part of it will seep through the structure into the $i$-th output channel. In the general case of spin-dependent interactions present in the system, $\hat{t}_{i j, \alpha}$ are potentially non-zero and the spin will rotate from its original orientation. Moreover, the magnitude of the transmitted flux can, in principle, now depend on the spin orientation of the incident flux.

Partially polarized electron fluxes can be mimicked by a number of independent (not phase coherent) elementary fluxes $\hat{u}_{q}$ through the same channel that are just additive in the case of our linear system

$$
F=\sum_{q} F_{q}, \boldsymbol{P} F=\sum_{q}(\boldsymbol{P} F)_{q}
$$

To present the unpolarized input flux in channel $j$, one can use, for example, two elementary fluxes $\hat{u}_{1}=(1,0)$ and $\hat{u}_{2}=(0,1)$. Now it is very easy to evaluate $F$ and $\boldsymbol{P}$ for the electron flux, transmitted into channel $i$. Indeed,

$$
F \propto\left|t_{i j, 1}\right|^{2}+\sum_{\alpha}\left|t_{i j, \alpha}\right|^{2}
$$

with the $\alpha$-component of spin polarization

$$
P_{\alpha} F \propto 2 \operatorname{Im}\left(t_{i j, 1} t_{i j, \alpha}^{*}+t_{i j, \alpha+1}^{*} t_{i j, \alpha+2}\right),
$$

where $x y z$ indices are cyclically permuted. ${ }^{11}$

There are strict fundamental limitations on the components of the scattering matrices. Hereafter we present an adaptation of these limitations suitable for the devices with the degenerate spin subchannels in the leads. ${ }^{10}$

With the total flux $\mathbf{F} \propto \mathbf{U}^{+} \mathbf{U}$, the requirement of flux conservation for $\mathbf{U}_{\text {out }}=\mathbf{S} \mathbf{U}_{\text {in }}$ corresponding to an arbitrary column $\mathbf{U}_{\text {in }}$ (symbolically representing coherent incident plane waves $\hat{u}_{i}$ coming through all channels), can only be guaranteed if

$$
\mathbf{S}^{+} \mathbf{S}=\mathbf{1}
$$

For a broad class of problems, including the one with intrinsic SO coupling, time-reversal invariance (with the operator $\hat{T}=-i \hat{\sigma}_{y} K$ where $K$ is the complex conjugation) establishes the following relation on the scattering matrix $\mathbf{S}$

$$
\hat{\sigma}_{y} \mathbf{S}^{*} \hat{\sigma}_{y} \mathbf{S}=\mathbf{1}
$$

This equation should be regarded as a symbolic one, with the Pauli matrix multiplications applied to each submatrix $\hat{r}_{i i}, \hat{t}_{i j}$ separately.

Combined with Eq. (6), this relation can be converted into a more practical form $\hat{\sigma}_{y} \mathbf{S}^{*} \hat{\sigma}_{y}=\mathbf{S}^{+}$, that immediately results in

$$
r_{i i, \alpha}=0, t_{i j, 1}=t_{j i, 1}, t_{i j, \alpha}=-t_{j i, \alpha},
$$

Thus, the polarization of the back-reflected flux from unpolarized source is not possible. ${ }^{12}$

Additional structure symmetry elements, when present, could provide further restricting relations on the components of $\mathbf{S}$ (see Ref. 10 for details). However, they are not necessary for the further consideration.

With all relevant basic quantities and concepts already introduced, it is now time to formulate and prove the following theorem: For an arbitrary multiterminal ballistic device with intrinsic $S O$ interaction that is connected to the normal metal contacts the equilibrium electron flux going out of the device through any particular terminal is unpolarized, i.e. ${ }^{13}$

$$
\sum_{j}(\boldsymbol{P} F)_{i j} \equiv 0
$$

We start the proof of this theorem with Eq. (6) and write out explicitly the $i i$-th matrix element of this equation

$$
\sum_{j}\left(\boldsymbol{S}^{+}\right)_{i j}(\boldsymbol{S})_{j i}=\hat{1}
$$

with

$$
\left(\boldsymbol{S}^{+}\right)_{i j}=\hat{t}_{j i}^{+}=\hat{1} t_{j i, 1}^{*}-i \sum_{\alpha} \hat{\sigma}_{\alpha} t_{j i, \alpha}^{*} .
$$

After performing summation over $i$ explicitly and collecting terms with $\hat{\sigma}_{\alpha}$ on the l.h.s. of the equation (no such terms present on the r.h.s.) we get

$$
\begin{array}{r}
\ldots+i \hat{\sigma}_{\alpha} \sum_{j \neq i}\left(t_{j i, 1}^{*} t_{j i, \alpha}-t_{j i, \alpha}^{*} t_{j i, 1}\right. \\
\left.+t_{j i, \alpha+1}^{*} t_{j i, \alpha+2}-t_{j i, \alpha+2}^{*} t_{j i, \alpha+1}\right)+\ldots
\end{array}
$$

Making use of Eq. (8), the equation under the sign of summation is exactly $2 \operatorname{Im}\left(t_{i j, 1} t_{i j, \alpha}^{*}+t_{i j, \alpha+1}^{*} t_{i j, \alpha+2}\right)$ which, according to Eq. (5) is an $\alpha$-polarized part of the flux $\left(P_{\alpha} F\right)_{i j}$ going out of the device through terminal $i$, induced by the unpolarized incident flux through terminal $j$ (and the incident electron fluxes are naturally unpolarized in case of normal metal terminals). Thus, it turns out that in order to satisfy the flux conservation condition of Eq. (6), $\sum_{j}(\boldsymbol{P} F)_{i j}$ should be zero, that concludes the proof.

Since this statement holds for any incident electron energy $E_{\text {kin }}$ independently, it holds, naturally, also after integration over all occupied states.

When the infinitesimal biases are applied to terminals, uncompensated nonequilibrium currents will flow 
through the device. In presence of SO interactions, these currents can generally become polarized (like, for example, it happens in the case of the T-shaped spin filter ${ }^{9}$ ). However, there are situations, when, as a consequence of the theorem proven above, these polarization processes are fundamentally prohibited.

(i) Two-terminal device.-An arbitrary system with just two connecting terminals [Fig. 1 b] cannot polarize transmitted electron flux (this finding was earlier announced in Ref. 10; see also Ref. 14 for the discussion of related phenomena). Indeed, the sum in Eq. (9) reduces in this case to a single term that is bound, by the theorem, to be zero.

(ii) $N$-terminal device with $N-1$ inputs connected in parallel.-When all but one terminal are kept at the same bias [Fig. 1 (c)], the output current through that last terminal is unpolarized. Again, for the $N$-th terminal we return exactly to the situation described by the theorem. For a not-so-obvious example, let us consider some ballistic device that is connected to the exterior by just two physical wires, one of them supporting multi-channel transport and another one allowing only a single propagating mode (apart from spin degeneracy): current in this second wire is always unpolarized.

In summary, we have formulated and proven a theorem relating very general and fundamental properties of a ballistic system (current conservation and the symmetry in respect to the time inversion) with the ability of this system to polarize transient currents. When all input currents are supplied by unpolarized sources, each and every output current is also unpolarized for the system in equilibrium, i.e., no equilibrium spin currents are allowed.

Acknowledgements.-This work was supported, in part, by DARPA, ONR, and SRC/MARCO.

${ }^{\star}$ kiselev@eos.ncsu.edu

${ }^{1}$ S. Murakami, N. Nagaosa, and S.-C. Zhang, Science 301, 1348 (2003).

${ }^{2}$ J. Sinova, D. Culcer, Q. Niu, N. A. Sinitsyn, T. Jungwirth, and A. H. MacDonald, cond-mat/0307663; Phys. Rev. Lett. 92, 126603 (2004).

${ }^{3}$ J. Schliemann and D. Loss, cond-mat/0310108; PRB 69, 165315 (2004); A. A. Burkov and A. H. MacDonald, condmat/0311328; J. Inoue, G. E. W. Bauer, and L. W. Molenkamp, cond-mat/0402442; Phys. Rev. B 70, 041303 (2004); O. V. Dimitrova, cond-mat/0405339 v.2; E. I. Rashba, cond-mat/0404723; E. G. Mishchenko, A. V. Shytov, and B. I. Halperin, cond-mat/0406730; A. Khaetskii, cond-mat/0408136; A. G. Mal'shukov and K. A. Chao, cond-mat/0410607.

${ }^{4}$ E. I. Rashba, cond-mat/0311110; Phys. Rev. B 68, 241315(R) (2003).
${ }^{5}$ A. G. Mal'shukov, C. S. Tang, C. S. Chu, and K. A. Chao, Phys. Rev. B 68, 233307 (2003).

${ }^{6}$ T. P. Pareek, Phys. Rev. Lett. 92, 076601 (2004).

${ }^{7}$ S. Souma and B. K. Nikolic, cond-mat/0410716.

${ }^{8} \mathrm{~S}$. Datta, Electronic transport in mesoscopic systems, (Cambridge University press, Cambridge, 1997), Ch. 3.

${ }^{9}$ A. A. Kiselev, K. W. Kim, Appl. Phys. Lett. 78, 775 (2001).

${ }^{10}$ A. A. Kiselev, K. W. Kim, cond-mat/0203261; J. Appl. Phys. 94, 4001 (2003).

11 This equation shows, in particular, that if only one component of $\hat{t}_{i j}$ is present, polarization of the transmitted electron flux is zero for unpolarized input.

${ }^{12}$ Note, that without the SO term the operator $K$ itself and any combination $\hat{\sigma}_{\alpha} K$ would commute with the Hamiltonian, so one would obtain $t_{i j, \alpha} \equiv 0$. This would indicate that there are no spin-polarizing effects in transmission as well, which is in agreement with common sense.

13 Because the back-scattered flux is always unpolarized, it does not matter whether the $i=j$ term is included into the summation or not.

${ }^{14}$ P. Bruno, Phys. Rev. Lett. 79, 4593 (1997); T. P. Pareek and P. Bruno, Phys. Rev. B 63, 165424 (2001); U. Zulicke and C. Schroll, Phys. Rev. Lett. 88, 029701 (2002).

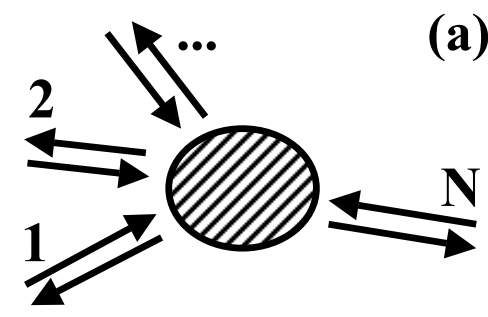

(b)
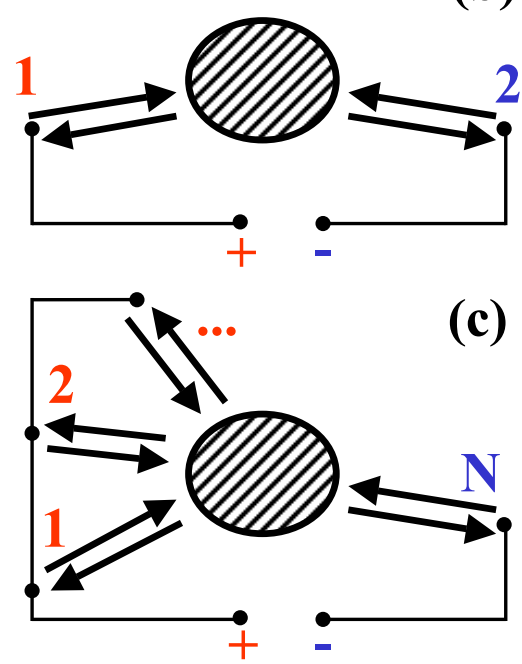

FIG. 1. (a) $N$-terminal ballistic device with intrinsic SO interaction (in shaded area). Arrows show direction of input and output fluxes through connecting terminals; (b) two-terminal device with bias applied; (c) $N$-terminal device with $N-1$ inputs connected in parallel. 